XX Міжнародний симпозіум «Методи дискретних особливостей в задачах математичної фізики/Discrete Singularities Methods in Mathematical Physics»,

УДК 519.14

МДОЗМФ/DSMMPh-2021

MSC 65C50

\title{
Gauss approximation for number distribution in of a Pascal's triangle
}

\author{
I.M. Abramov, G.S. Abramov
}

Kherson National Technical University, Kherson State Maritime Academy, Kherson, Ukraine

E-mail: gennadabra@gmail.com,zealot613@gmail.com

\begin{abstract}
We received normal distribution parameters that approximates the distribution of numbers in the n-th row of Pascal's triangle. We calculated the values for normalized moments of even orders and shown their asymptotic tendency towards values corresponding to a normal distribution. We have received highly accurate approximations for central elements of even rows of Pascal's triangle, which allows for calculation of binomial, as well as trinomial (or, in general cases, multinomial) coefficients. A hypothesis is proposed, according to which it is possible that physical and physics-chemical processes function according to Pascal's distribution, but due to how slight its deviation is from a normal distribution, it is difficult to notice. It is also possible that as technology and experimental methodology improves, this difference will become noticeable where it is traditionally considered that a normal distribution is taking place.
\end{abstract}

Key words: Pascal's triangle, binomial coefficients, normal (Gaussian) distribution, trinomial coefficients, multinomial coefficients.

\section{Распределение чисел в строках треугольника Паскаля и его гауссовская аппроксимация}

\section{И.М. Абрамов, Г.С. Абрамов}

Херсонский национальный технический университет, Херсонская государственная морская академия, Херсон, Украина

E-mail: gennadabra@gmail.com,zealot613@gmail.com

\begin{abstract}
Получены значения параметров нормального распределения, аппроксимирующего распределение чисел в n-ой строке треугольника Паскаля. Вычислены значения нормированных моментов чётных порядков и показано их асимптотическое приближение к значениям, соответствующим нормальному распределению. Получены высокоточные приближения для центральных элементов чётных строк треугольника Паскаля, что даёт возможность вычислять биномиальные, а также триномиальные (и, в общем случае, мультиномиальные) коэффициенты. Выдвинута гипотеза, согласно которой, возможно, в некоторых физических или физико-химических процессах работает именно распределение Паскаля, но в связи с незначительным отклонением данного распределения от нормального, его очень непросто заметить. Возможно также, что при усовершенствовании техники и методического обеспечения эксперимента, это различие окажется заметным там, где традиционно считается, что имеет место нормальное распределение.
\end{abstract}

Ключевые слова: треугольник Паскаля, биномиальные коэффициенты, нормальное (гауссовское) распределение, триномиальные коэффициенты, мультиномиальные коэффициенты.

(С) Абрамов И.М., Абрамов Г.С., 2021 


\section{1. Постановка проблемы}

Известно, что треугольник Паскаля составляют так называемые биномиальные коэффициенты. Биномиальные и триномиальные коэффициенты часто встречаются при решении комбинаторных, математических и физических задач, а также в различных технических приложениях. Так, во многих задачах физической и химической кинетики, при исследовании вероятностных процессов, а также в задачах теории информации часто возникает необходимость вычислять различные конфигурации на множествах, в частности числа сочетаний $\mathrm{C}_{\mathrm{n}}^{\mathrm{m}}$, то есть биномиальные коэффициенты. Чаще всего в этих случаях для вычисления факториалов используют известную приближённую формулу Стирлинга, которая даёт достаточно грубое приближение. Поэтому задача отыскания более точного приближения является актуальной. Известно также, что асимптотикой биномиального распределения является нормальное (гауссовское) распределение. Однако оно реализуется с хорошей точностью лишь для достаточно больших номеров строк, в пределе при $n \rightarrow \infty$. Конкретные же сведения о «кинетике» этого приближения; о том, каким образом изменяются числовые характеристики аппроксимирующего нормального распределения при увеличении номера строки, отсутствуют.

\section{2. Анализ публикаций}

Треугольнику Паскаля посвящена довольно обширная научная литература [310], в которой выделяются две фундаментальные монографии [3, 4], где биномиальным коэффициентам посвящены отдельные главы. В книге Р. Грэхема, Д. Кнута и О. Паташника «Конкретная математика. Основание информатики» [3] в главе «Биномиальные коэффициенты» рассматриваются доказательства многочисленных тождеств (часто совершенно удивительных) и оригинальные способы вычисления различных частных сумм. В монографии В. Феллера «Введение в теорию вероятностей и её приложения» [4] рассматривается нормальное приближение для биномиального распределения, однако погрешность результатов (1-2\%) нельзя признать удовлетворительной.

\section{3. Формулировка целей исследования}

Целями данной работы, являющейся продолжением исследований, начатых в работах $[1,2]$, было рассмотрение асимптотики приближения распределения чисел в строках треугольника Паскаля к нормальному; получение высокоточных приближений для центральных элементов строк треугольника Паскаля и их использование для вычисления триномиальных (мультиномиальных) коэффициентов; рассмотрение отклонений плотности паскалевского распределения от гауссовского в окрестности центральных элементов.

4. Аппроксимация распределения чисел в треугольнике Паскаля к гауссовскому

В предыдущей работе авторов [1], целью которой было установление связи распределения чисел в строках треугольника Паскаля с нормальным (гауссовским) распределением, были решены следующие задачи: определены числовые характеристики нормального приближения для распределения чисел в строках треугольника Паскаля; выявлены зависимости этих параметров от номера строки; выполнено сравнение распределения чисел в строках треугольника Паскаля с нормальным приближением и рассмотрена асимптотика приближения паскалевского распределения к нормальному при увеличении номера строки. 
XX Міжнародний симпозіум «Методи дискретних особливостей в задачах математичної фізики/Discrete Singularities Methods in Mathematical Physics»,

\section{МДОЗМФ/DSMMPh-2021}

Математическое ожидание и дисперсию распределения чисел в строках треугольника Паскаля вычисляли следующим образом:

$$
\bar{x}=N^{-1} \sum_{i=1}^{k} x_{i} n_{i} ; \quad S^{2}=N^{-1} \sum_{i=1}^{k} x_{i}^{2} n_{i}-\bar{x}^{2},
$$

где $x_{i}$ - номер позиции (элемента) в строке треугольника Паскаля; $n_{i}-$ значение элемента на $i$-ой позиции в строке треугольника Паскаля; $k=n+1-$ количество элементов в $n$-ой строке треугольника Паскаля; $N$ - сумма всех элементов в данной строке треугольника Паскаля: $N=2^{n}$.

Математическое ожидание, дисперсия и среднеквадратичное отклонение от номера строки треугольника Паскаля зависит следующим образом:

$$
\bar{x}=\frac{n+2}{2} ; \quad S^{2}=\frac{n}{4} ; \quad S=\frac{\sqrt{n}}{2} .
$$

Для соответствующих значений матожидания и среднеквадратичного отклонения построены гауссовские распределения и выполнено сравнение полученных гауссовских кривых с фактическим частотным распределением в соответствующих строка треугольника Паскаля. Для этого были вычислены относительные частоты распределения чисел в строках треугольника Паскаля (значение данного элемента строки делилось на сумму всех чисел в строке $-2^{n}$ ).

На рис. 1 приведены соответствующие эмпирические распределения (основанные на вычисленных частотах) в сравнении с теоретическими распределениями (гауссовские кривые). Видно, что с увеличением номера строки распределение чисел в строках треугольника Паскаля приближается к нормальному распределению. Это даёт основание считать, что нормальное приближение является хорошей аппроксимацией распределения в $n$-той строке треугольника Паскаля (очевидно, тем лучшей, чем больше номер строки $n$ ):

$$
x_{n} \in \mathrm{N}\left(\frac{n+2}{2} ; \frac{\sqrt{n}}{2}\right) .
$$

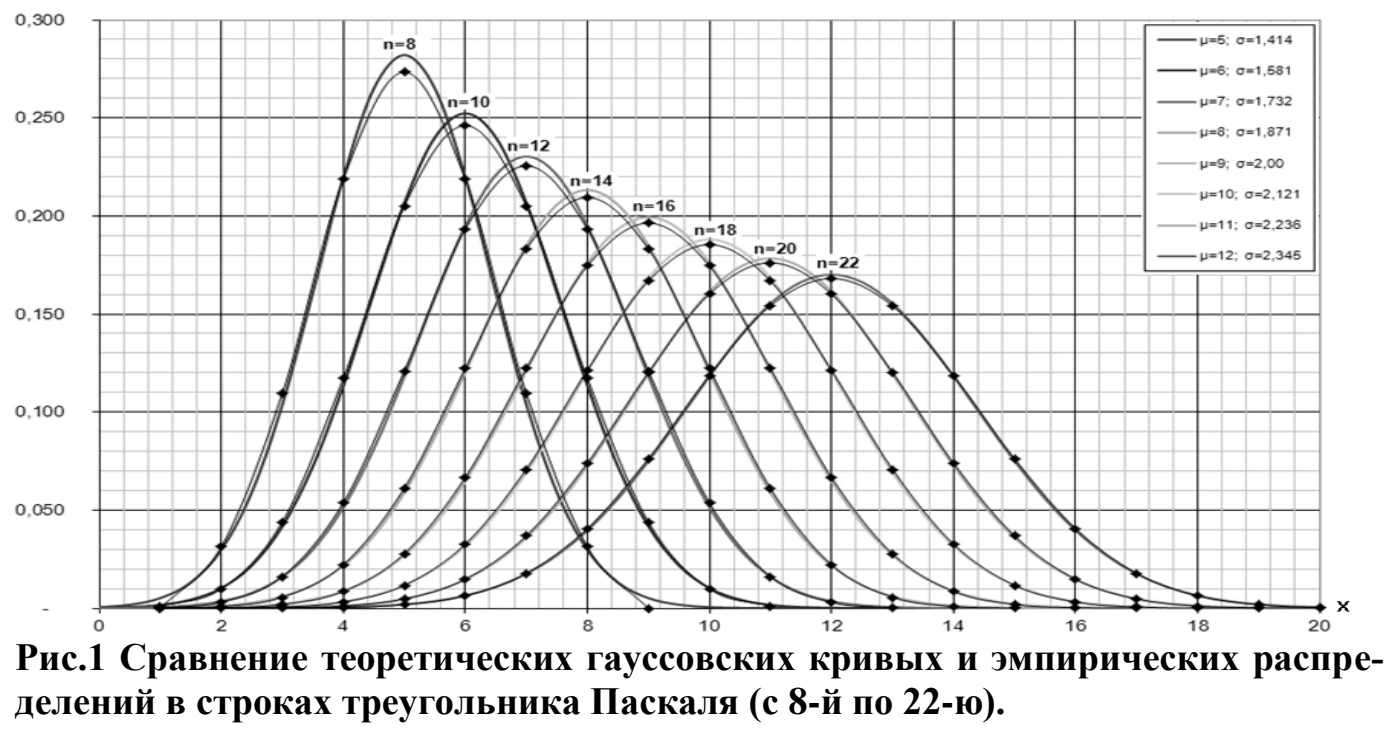


На рис. 1 видны различия между распределением в строке треугольника Паскаля и нормальным распределением (которое тем заметней, чем меньше номер строки): максимальное значение распределений в строках треугольника Паскаля чуть меньше максимума на соответствующих гауссовских кривых.

Известно, что искажения нормального распределения связаны с моментами третьего и четвёртого порядков (соответствующие коэффициенты называются асимметрией и эксцессом). Асимметрия, описывающая искажения гауссовской кривой вдоль оси абсцисс, в нашем случае отсутствует, в силу симметричности чисел в строках треугольника Паскаля. (Отсюда следует, что все моменты нечётных порядков для строк треугольника Паскаля равны нулю). Следовательно, для описания наблюдаемых различий паскалевского распределения от гауссовского приближения, необходимо вычислять значения эксцесса (связанного с моментом четвёртого порядка), и, вообще говоря, моменты чётных порядков (4-го, 6-го, 8-го и т. д.). Очевидно, что вычисляемые значения эксцесса должны быть отрицательными (т. к. «уплощение» гауссовской кривой характеризуется отрицательным значением эксцесса), при этом, с возрастанием номера строки значение эксцесса должно асимптотически приближаться к нулю.

Эксцесс $\left(E_{x}\right)$ вычисляли с помощью центрального момента 4-го порядка - $\mu^{4}$ :

$$
\mu^{4}=\frac{\sum_{i=1}^{n+1}\left(x_{i}-\bar{x}\right)^{4} n_{i}}{\sum_{i=1}^{n+1} n_{i}}=\frac{\sum_{i=1}^{n+1}\left(x_{i}-\bar{x}\right)^{4} n_{i}}{2^{n}}, \quad E_{x}=\frac{\mu^{4}}{\sigma^{4}}-3 .
$$

Значения эксцесса отрицательны и с увеличением номера строки асимптотически приближаются к нулю, что свидетельствует о приближении распределения в строках с большим номером к гауссовскому.

Плотность аппроксимирующего гауссовского распределения в $n$-ой строке треугольника имеет следующий вид:

$$
f_{n}(x) \approx \frac{2}{\sqrt{2 \pi n}} \exp \left(-\frac{\left(x-\frac{n+2}{2}\right)^{2}}{\frac{2 n}{4}}\right)=\sqrt{\frac{2}{\pi n}} \exp \left(-\frac{2\left(x-\frac{n+2}{2}\right)^{2}}{n}\right) .
$$

В работе [1] с использованием данной формулы проведены приближённые вычисления элементов строк треугольника Паскаля. Число сочетаний $\mathrm{C}_{\mathrm{n}}^{\mathrm{m}}$ в нашем случае является $(\mathrm{m}+1)$-м элементом n-ой строки треугольника (т. к. отсчёт величины $x$ мы начали не с нуля, а с единицы). Тогда число сочетаний можно вычислить по следующей приближённой формуле:

$$
C_{n}^{m} \approx 2^{n} \sqrt{\frac{2}{\pi n}} \exp \left(-\frac{(2 m-n)^{2}}{2 n}\right) .
$$

Проще всего с помощью этой формулы вычисляется значение центральных элементов чётных строк треугольника, когда $m=n / 2$ :

$$
C_{n}^{m=\frac{n}{2}} \approx \frac{2^{n}}{\sqrt{\pi m}} \text { или } C_{2 n}^{n} \approx \frac{4^{n}}{\sqrt{\pi n}} .
$$

Можно показать, что приближение (1) соответствует вычислению числа сочетаний, если факториалы вычислять по формуле Стирлинга: 
XX Міжнародний симпозіум «Методи дискретних особливостей в задачах математичної фізики/Discrete Singularities Methods in Mathematical Physics»,

МДОЗМФ/DSMМPh-2021

$$
n ! \approx \sqrt{2 \pi n}\left(\frac{n}{e}\right)^{n} .
$$

В работе [1] проведено также сравнение точных значений центральных элементов чётных строк треугольника и их приближённых значений, вычисленных с помощью гауссовской аппроксимации (табл. 1):

Таблица 1. Сравнение точных значений и гауссовской оценки центральных элементов чётных строк

\begin{tabular}{|l|l|l|l|}
\hline $\begin{array}{l}\text { Номер } \\
\text { строки } \\
\mathrm{n}\end{array}$ & $\begin{array}{l}\text { Точное значение } \\
C_{n}^{m=\frac{n}{2}}=\frac{n !}{(m !)^{2}}\end{array}$ & $\begin{array}{l}\text { Приближённое значение } \\
C_{n}^{m=\frac{n}{2}} \approx \frac{2^{n}}{\sqrt{\pi m}}\end{array}$ & $\begin{array}{l}\text { Относительная } \\
\text { ошибка } \varepsilon, \%\end{array}$ \\
\hline 30 & $1,551175 \cdot 10^{8}$ & $1,564153 \cdot 10^{8}$ & 0,84 \\
\hline 50 & $1,264106 \cdot 10^{14}$ & $1,270442 \cdot 10^{14}$ & 0,50 \\
\hline 60 & $1,182646 \cdot 10^{17}$ & $1,187583 \cdot 10^{17}$ & 0,42 \\
\hline 100 & $1,008913 \cdot 10^{29}$ & $1,011439 \cdot 10^{29}$ & 0,25 \\
\hline 200 & $9,054851 \cdot 10^{58}$ & $9,066177 \cdot 10^{58}$ & 0,125 \\
\hline 400 & $1,029525 \cdot 10^{119}$ & $1,030169 \cdot 10^{119}$ & 0,0625 \\
\hline 800 & $1,88042442 \cdot 10^{239}$ & $1,88101214 \cdot 10^{239}$ & 0,03125 \\
\hline
\end{tabular}

Из данных табл. 1 следует, что относительная ошибка (разность между приближённым и точным значением, отнесенная к точному значению) убывает с возрастанием номера строки по обратно пропорциональной зависимости, что позволяет записать выражение для относительной ошибки:

$$
\frac{\frac{2^{n}}{\sqrt{\pi m}}-C_{n}^{m=n / 2}}{C_{n}^{m=n / 2}}=\frac{0,25}{n},
$$

откуда следует более точное приближение:

$$
C_{n}^{m=n / 2} \approx \frac{2^{n}}{\sqrt{\pi m}} \cdot \frac{n}{n+0,25} .
$$

Сравним точное значение с полученным приближением (табл. 2):

Таблица 2. Сравнение точных значений и приближения (6) для центральных элементов чётных строк треугольника Паскаля

\begin{tabular}{|l|l|l|l|}
\hline $\begin{array}{l}\text { Номер } \\
\text { строки } n\end{array}$ & $\begin{array}{c}\text { Точное значение } \\
C_{n}^{m=\frac{n}{2}}=\frac{n !}{(m !)^{2}}\end{array}$ & $\begin{array}{l}\text { Приближённое значение (6) } \\
C_{n}^{m=n / 2} \approx \frac{2^{n}}{\sqrt{\pi m}} \cdot \frac{n}{n+0,25}\end{array}$ & $\begin{array}{l}\text { Относительная } \\
\text { ошибка } \varepsilon_{1}, \%\end{array}$ \\
\hline 30 & $1,551175 \cdot 10^{8}$ & $1,551226 \cdot 10^{8}$ & 0,0033 \\
\hline 50 & $1,264106 \cdot 10^{14}$ & $1,264121 \cdot 10^{14}$ & 0,0012 \\
\hline 60 & $1,182646 \cdot 10^{17}$ & $1,182655 \cdot 10^{17}$ & 0,0008 \\
\hline 100 & $1,0089134 \cdot 10^{29}$ & $1,0089166 \cdot 10^{29}$ & 0,0003 \\
\hline 200 & $9,0548515 \cdot 10^{58}$ & $9,0548578 \cdot 10^{58}$ & 0,00007 \\
\hline 400 & $1,029525 \cdot 10^{119}$ & $1,029525 \cdot 10^{119}$ & \multirow{2}{*}{$\varepsilon_{1}=\frac{3}{n^{2}} \%$} \\
\hline 800 & $1,88042442 \cdot 10^{239}$ & $1,88042442 \cdot 10^{239}$ & \\
\hline
\end{tabular}


Видно, что относительная ошибка этого приближения $\varepsilon_{1}$ обратно пропорциональна квадрату номера строки, что даёт возможность сформулировать ещё более точное приближение:

$$
C_{n}^{m=n / 2} \approx \frac{2^{n}}{\sqrt{\pi m}} \cdot \frac{n}{n+0,25} \cdot \frac{n^{2}}{n^{2}+0,03}
$$

или

$$
C_{2 n}^{n} \approx \frac{4^{n}}{\sqrt{\pi n}} \cdot \frac{n}{n+0,125} \cdot \frac{n^{2}}{n^{2}+0,0075} .
$$

Точность этого приближения настолько высока, что даже для небольших $n$ она даёт очень хорошую оценку. Приближение можно записать в виде:

$$
C_{2 n}^{n} \approx \frac{4^{n}}{\sqrt{\pi n}} \cdot\left(\frac{1}{1+\frac{0,125}{n}+\frac{0,0075}{n^{2}}+\frac{0,0009375}{n^{3}}}\right) .
$$

Имея достаточно точные значения центрального элемента чётной строки треугольника Паскаля можно легко вычислить и остальные элементы строки:

$$
C_{2 n}^{n \pm k}=C_{2 n}^{n} \cdot \frac{n}{n+1} \cdot \frac{n-1}{n+2} \cdot \ldots \cdot \frac{n+1-k}{n+k},
$$

или, в более компактной форме,

$$
C_{2 n}^{n \pm k}=C_{2 n}^{n} \prod_{i=1}^{k} \frac{n+1-i}{n+i}
$$

Из полученных выражений следует рекуррентная формула для вычисления факториалов:

$$
(2 n) !=\frac{4^{n}(n !)^{2}}{\sqrt{\pi n}} \cdot \frac{n}{n+0,125} \cdot \frac{n^{2}}{n^{2}+0,0075}
$$

или

$$
(2 n) !=\frac{4^{n}(n !)^{2}}{\sqrt{\pi n}} \cdot\left(\frac{1}{1+\frac{0,125}{n}+\frac{0,0075}{n^{2}}+\frac{0,0009375}{n^{3}}}\right) .
$$

Следуя [3], покажем, что с помощью биномиальных коэффициентов можно вычислить триномиальные коэффициенты, а именно: триномиальные коэффициенты могут быть выражены через произведение биномиальных коэффициентов.

$$
C_{n}^{m} C_{m}^{k}=\frac{n !}{m !(n-m) !} \cdot \frac{m !}{k !(m-k) !}=\frac{n !}{k !(m-k) !(n-m) !} .
$$

Переобозначив переменные, получим:

$$
C_{n}^{m} C_{m}^{k}=\frac{(a+b+c) !}{a ! b ! c !}
$$

где $\mathrm{n}=a+b+c ; k=a ; b=m-k ; c=n-m$. 
XX Міжнародний симпозіум «Методи дискретних особливостей в задачах математичної фізики/Discrete Singularities Methods in Mathematical Physics»,

\section{МДОЗМФ/DSMМPh-2021}

Таким образом, произведение биномиальных коэффициентов $C_{n}^{m} C_{m}^{k}$ есть не что иное, как триномиальный коэффициент, который появляется в триномиальной теореме:

$$
(x+y+z)^{n}=\sum_{\substack{0 \leq a, b, c \leq n \\ a+b+c=n}} \frac{(a+b+c) !}{a ! b ! c !} x^{a} y^{b} z^{c}=\sum_{\substack{0 \leq a, b, c \leq n \\ a+b+c=n}} C_{n}^{b+a} C_{\mathrm{b}+\mathrm{a}}^{a} x^{a} y^{b} z^{c} .
$$

Учитывая следующее равенство для произведений биномиальных коэффициентов

$$
C_{n}^{m} C_{m}^{k}=\frac{n !}{k !(m-k) !(n-m) !}=\frac{n !}{k !(n-k) !} \cdot \frac{(n-k) !}{(m-k) !(n-m) !}=C_{n}^{k} C_{n-k}^{m-k},
$$

триномиальные коэффициенты можно представить в другой форме, несколько более удобной для суммирования:

$$
(x+y+z)^{n}=\sum_{\substack{0 \leq a, b, c \leq n \\ a+b+c=n}} \frac{(a+b+c) !}{a ! b ! c !} x^{a} y^{b} z^{c}=\sum_{\substack{0 \leq a, b, c \leq n \\ a+b+c=n}} C_{n}^{a} C_{b+c}^{b} x^{a} y^{b} z^{c} .
$$

Обобщением биномиальных и триномиальных коэффициентов служат мультиномиальные коэффициенты, которые также могут быть представлены в виде произведения соответствующего количества биномиальных коэффициентов:

$$
\frac{\left(a_{1}+a_{2}+\ldots+a_{m}\right) !}{a_{1} ! a_{2} ! \ldots a_{m} !}=C_{a_{1}+a_{2}+\ldots+a_{m}}^{a_{2}+\ldots} C_{a_{m-1}+a_{m}}^{a_{m}} .
$$

Таким образом, имея достаточно точное выражение для биномиальных коэффициентов можно вычислять и триномиальные коэффициенты и, в общем случае, мультиномиальные коэффициенты.

Используя предлагаемое нами более точное приближение для биномиальных коэффициентов можно значительно повысить точность получаемых результатов, там, где раньше вынуждены были ограничиваться весьма приближёнными, как при использовании формулы Стирлинга, которая, как мы показали, даёт достаточно грубое приближение.

Рассмотрим более детально отклонения распределения чисел в строках треугольника Паскаля от аппроксимирующего гауссовского распределения в окрестности центральных элементов.

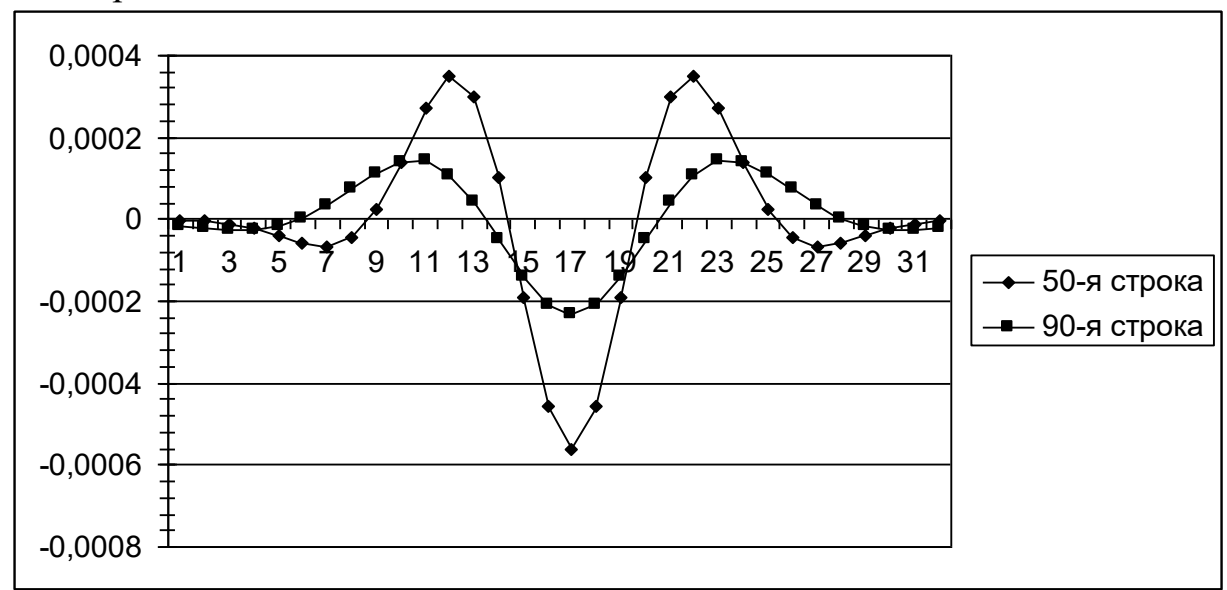


Рис. 2 Графики отклонений значений плотности распределений в строках треугольника Паскаля от нормального распределения в окрестности центральных элементов для 50-й и 90-й строк

На рис. 2 представлены графики отклонений значений плотности распределений в строках треугольника Паскаля от нормального распределения в окрестности центральных элементов для 50-й и 90-й строк. Видно, что если для центральных элементов строк эта разность отрицательна (паскалевское распределение «приплюснуто» относительно гауссовского), то в ближайшей окрестности наблюдается обратная ситуация - плотность паскалевского распределения больше, чем плотность гауссовского распределения. Дальше, с удалением от центральных элементов снова наблюдается отрицательная разность, однако этот «волнообразный» процесс быстро затухает и разность асимптотически приближается к нулю. Видно также, что для строки с более высоким номером амплитуда колебаний меньше по сравнению с распределением в строке с меньшим номером, то есть с возрастанием номера строки идёт сглаживание (затухание) колебаний плотности паскалевского распределения относительно гауссовского и с точки зрения их амплитуды.

На рис. 3 представлен график отклонений плотности распределений в строках треугольника Паскаля для n от 10 до 20 включительно.

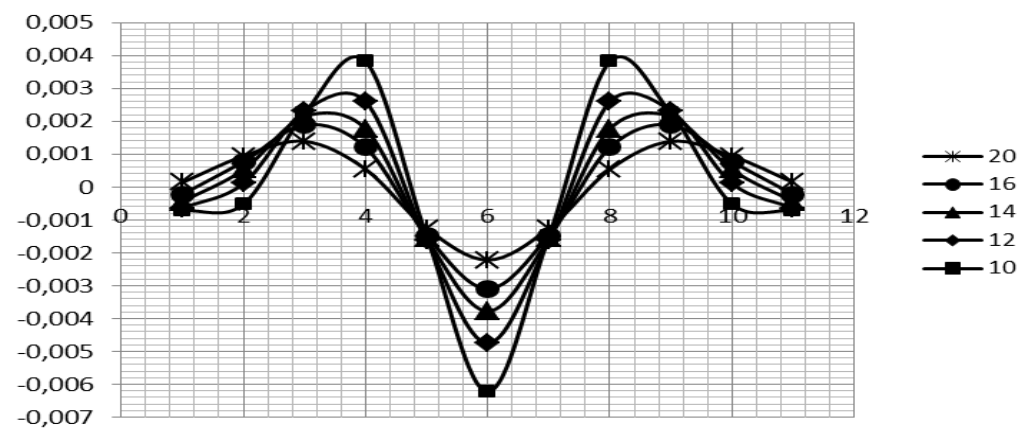

Рис. 3 Графики отклонений значений плотности распределений в строках треугольника Паскаля от нормального распределения в окрестности центральных элементов для строк от 10-й до 20-й.

В работе выполнено численное интегрирование (методом Симпсона) плотности распределения в строках треугольника Паскаля. Для строк с номерами $\mathrm{n}=50$ и n=90 получены значения, которые с высокой точностью можно считать единицей (0,9999998), следовательно, распределение в этих строках треугольника Паскаля удовлетворяет условию нормировки на единицу, которое предъявляется к плотности распределения $\left(\int_{-\infty}^{+\infty} f(x) d x=1\right)$. В связи с этим можно предложить следующую гипотезу: возможно, в некоторых тонких физических или физикохимических вероятностных процессах работает именно распределение Паскаля, но поскольку различие между паскалевским и его аппроксимирующим гауссовским распределением в абсолютном значении очень мало (от 0,0001 до 0,01), то это различие (волнообразное отклонение в окрестностях иеетральных элементов), в соответствующих экспериментах может быть незаметным. Очевидно, в реальных экспериментах значения плотности распределения определяются со значительно большей погрешностью, чем 0,0001, и даже 0,001. Однако, отклонения 
XX Міжнародний симпозіум «Методи дискретних особливостей в задачах математичної фізики/Discrete Singularities Methods in Mathematical Physics», МДОЗМФ/DSMМPh-2021

порядка 0,01 уже могут быть замечены: если бы усовершенствование техники и методического обеспечения эксперимента позволили бы иметь погрешность на уровне указанной, то, возможно, в некоторых тонких вероятностных экспериментах удалось бы заметить отклонение от гауссовской кривой и показать, что, имеет место именно распределение Паскаля там, где традиционно считается, что работает нормальное распределение.

Из рис. 3 видно, что волнообразный процесс в окрестности центральных элементов для строк с небольшим номером характеризуется большей амплитудой: если на рис. 2 это различие в четвертом знаке после запятой, то на рис. 3 - различие в сто раз больше, что соответственно увеличивает шансы его экспериментального выявления. Именно на этой основе можно осуществлять поиск отклонений от гауссовского распределения на выборках с относительно небольшой статистикой, где наблюдаются отрицательные значения эксцесса (а также других моментов чётных порядков).

\section{5. Выводы}

Проведены вычисления числовых характеристик распределения чисел в строках треугольника Паскаля; осуществлено сравнение гауссовской аппроксимации и эмпирических распределений; приведена формула для плотности распределения в nой строке треугольника Паскаля.

На основе гауссовской аппроксимации получены высокоточные приближения для вычисления значений центральных элементов чётных строк треугольника Паскаля. Достаточно точные значения биномиальных коэффициентов позволяют вычислять триномиальные коэффициенты (с помощью произведения пар биномиальных коэффициентов) и, в общем случае, мультиномиальные коэффициенты (как произведения соответствующего числа биномиальных коэффициентов).

Исследованы отклонения значений плотности распределений в строках треугольника Паскаля от нормального распределения в окрестности центральных элементов. Показан их волнообразный характер и процесс асимптотического сглаживания колебаний плотности при увеличении номера строки треугольника Паскаля, который свидетельствует о приближении к гауссовскому распределению.

Выдвинута гипотеза, согласно которой, возможно, в некоторых физических или физико-химических процессах работает именно распределение Паскаля, но в связи с незначительным отклонением данного распределения от нормального, его очень непросто заметить. Возможно также, что при усовершенствовании техники и методического обеспечения эксперимента, это различие окажется заметным там, где традиционно считается, что имеет место нормальное распределение.

\section{ЛИТЕРАТУРА}

1. Abramov G.S., Abramov I.M. Normalnoe priblizhenie dlya raspredeleniya chisel v strokah treugolnika Paskalya. Vestnik HNTU. 2014. 3(50). P. 185-191.

2. Abramov G.S., Abramov I.M. Gaussovskaya approksimatsiya dlya raspredeleniya chisel v strokah treugolnika Paskalya. Vestnik HNTU. 2015. 3(54). P. 319-325.

3. Grehem R., Knut D., Patashnik O. Konkretnaya matematika. Osnovanie informatiki. M.: Mir, 1998. 703 p.

4. Feller V. Vvedenie v teoriyu veroyatnostey i eyo prilozheniya. M.: Mir, 1984. 528 p. 
5. Bondarenko B.A. Obobschyonnyie treugolniki i piramidyi Paskalya, ih fraktalyi, grafyi i prilozheniya. Tashkent, 1990. $192 \mathrm{p}$.

6. Kuzmin O.V. Nekotoryie kombinatornyie chisla $v$ obobschyonnoy piramide Paskalya. In: Asimptoticheskie i perechislitelnyie zadachi kombinatornogo analiza. Irkutsk: Izdatelstvo Irkutskogo universiteta, 1998. p. 90-100.

7. Kuzmin O.V. Obobschennyie piramidyi Paskalya i ih prilozheniya. Novosibirsk, 2000. $64 \mathrm{p}$

8. Uspenskiy V.A. Treugolnik Paskalya. M.: Nauka, 1979. 48 p.

9. Enzenzberger H.M. Duh chisla. Kharkov: Knizhnyiy klub «Klub simeynogo dozvillya», 2002. $272 \mathrm{p}$.

10. Gardner M. Matematicheskie novellyi. M.: Mir, 1974. 456 p.

Надійшла 21.05.2021

\section{Розподіл чисел у рядках трикутника Паскаля та його гаусівська апроксимація}

\section{I.М. Абрамов, Г.С. Абрамов}

Херсонський національний технічний університет, Херсонска державна морська академія,

Херсон, Україна

E-mail:gennadabra@gmail.com,zealot613@gmail.com

Отримано значення параметрів нормального розподілу, апроксимируючого розподіл чисел в n-ому рядку трикутника Паскаля. Обчислено значення нормованих моментів парних порядків і показано їх асимптотичне наближення до значень, відповідним нормальному розподілу. Отримано високоточні наближення для центральних елементів парних рядків трикутника Паскаля, що дає можливість обчислювати біноміальні, а також триноміальні (i, в загальному випадку, мультиноміальні) коефіцієнти. Висунуто гіпотезу, згідно з якою, можливо, в деяких фізичних або фізико-хімічних процесах працює саме розподіл Паскаля, але в зв'язку з незначним відхиленням даного розподілу від нормального, його дуже непросто помітити. Можливо також, що при удосконаленні техніки і методичного забезпечення експерименту, ця різниця виявиться помітною там, де традиційно вважається, що має місце нормальний розподіл.

Ключові слова: трикутник Паскаля, біноміальні коефіцієнти, нормальний (гаусівський) розподіл, триноміальні коефіцієнти, мультиноміальні коефіцієнти. 